\title{
AXIOMATIZATIONS AND FACTORIZATIONS OF SUGENO UTILITY FUNCTIONS
}

\author{
MIGUEL COUCEIRO AND TAMÁS WALDHAUSER
}

\begin{abstract}
In this paper we consider a multicriteria aggregation model where local utility functions of different sorts are aggregated using Sugeno integrals, and which we refer to as Sugeno utility functions. We propose a general approach to study such functions via the notion of pseudo-Sugeno integral (or, equivalently, pseudo-polynomial function), which naturally generalizes that of Sugeno integral, and provide several axiomatizations for this class of functions.

Moreover, we address and solve the problem of factorizing a Sugeno utility function as a composition $q\left(\varphi_{1}\left(x_{1}\right), \ldots, \varphi_{n}\left(x_{n}\right)\right)$ of a Sugeno integral $q$ with local utility functions $\varphi_{i}$, if such a factorization exists.
\end{abstract}

\section{INTRODUCTION}

The importance of aggregation functions is made apparent by their wide use, not only in pure mathematics (e.g., in the theory of functional equations, measure and integration theory), but also in several applied fields such as operations research, computer and information sciences, economics and social sciences, as well as in other experimental areas of physics and natural sciences. For general background, see [1, 17] and for a recent reference, see [16].

In many applications, the values to be aggregated are first to be transformed by mappings $\varphi_{i}: X_{i} \rightarrow Y, i=1, \ldots, n$, so that the transformed values (which are usually real numbers) can be aggregated in a meaningful way by a function $M: Y^{n} \rightarrow Y$. The resulting composed function $U: X_{1} \times \cdots \times X_{n} \rightarrow Y$ is then defined by

$$
U\left(x_{1}, \ldots, x_{n}\right)=M\left(\varphi_{1}\left(x_{1}\right), \ldots, \varphi_{n}\left(x_{n}\right)\right) .
$$

Such an aggregation model is used for instance in multicriteria decision making where the criteria are not commensurable. Here each $\varphi_{i}$ is a local utility function, i.e., orderpreserving mapping, and the resulting function $U$ is referred to as an overall utility function (also called global preference function). For general background see 3 .

In this paper, we consider this aggregation model in a purely ordinal decision setting, where $Y$ and each $X_{i}$ are bounded chains $L$ and $L_{i}$, respectively, and where $M: L^{n} \rightarrow L$ is a Sugeno integral [11, 21, 22] or, more generally, a lattice polynomial function. We refer to the resulting compositions as pseudo-Sugeno integrals and pseudo-polynomial functions, respectively. The particular case when each $L_{i}$ is the same chain $L^{\prime}$, and each $\varphi_{i}$ is the same mapping $\varphi: L^{\prime} \rightarrow L$, was studied in 8 where the corresponding compositions $U=M \circ \varphi$ were called quasi-Sugeno integrals and quasi-polynomial functions. Such mappings were characterized as solutions of certain functional equations and in terms of necessary and sufficient conditions which have natural interpretations in decision making and aggregation theory.

Here, we take a similar approach and study pseudo-Sugeno integrals from an axiomatic point of view, and seek necessary and sufficient conditions for a given function to be factorizable as a composition of a Sugeno integral with unary maps.

Key words and phrases. Pseudo-Sugeno integral, pseudo-polynomial function, local utility function, overall utility function, Sugeno utility function, axiomatization. 
The importance of such an axiomatization is attested by the fact that this framework subsumes the Sugeno utility model. Since overall utility functions (1) where $M$ is a Sugeno integral, coincide exactly with order-preserving pseudo-Sugeno integrals (see Corollary 2, we are particularly interested in the case when the inner mappings $\varphi_{i}$ are local utility functions.

As mentioned, this aggregation model is deeply rooted in multicriteria decision making, where the variables $x_{i}$ represent different properties of the alternatives (e.g., price, speed, safety, comfort level of a car), and the overall utility function assigns a score to the alternatives that helps the decision maker to choose the best one (e.g., to choose the car to buy). A similar situation is that of subjective evaluation: 3. $f$ outputs the overall rating of a certain product by customers, and the variables $x_{i}$ represent the various properties of that product. The way in which these properties influence the overall rating can give information about the attitude of the customers. A factorization of the (empirically) given overall utility function $f$ in the form (1) can be used for such an analysis; this is our main motivation to also address this problem.

The paper is organized as follows. In Section 2 we recall the basic definitions and terminology, as well as the necessary results concerning polynomial functions (and, in particular, Sugeno integrals) used in the sequel. In Section 3 , we focus on pseudoSugeno integrals as a tool to study certain overall utility functions. We introduce the notion of pseudo-polynomial function in Subsection 3.1 and show that, even though seemingly more general, it can be equivalently defined in terms of Sugeno integrals. An axiomatization of this class of generalized polynomial functions is given in Subsection 3.2. Sugeno utility functions are introduced in Subsection 3.3. as certain order-preserving pseudo-Sugeno integrals, and then characterized in Subsection 3.4 by means of necessary and sufficient conditions which extend well-known properties in aggregation function theory. Within this general setting for studying Sugeno utility functions, it is natural to consider the inverse problem which asks for factorizations of a Sugeno utility function as a composition of a Sugeno integral with local utility functions. This question is addressed in Section 4 where an algorithmic procedure is provided for constructing these factorizations of Sugeno utility functions. We present the algorithm in Subsection 4.1. which is illustrated by a concrete example in Subsection 4.2, and in Subsection 4.3 we show that this procedure does indeed produce the desired factorizations.

This manuscript is an extended version of, 9, 10 whose results were presented at the conference MDAI 2010.

\section{Lattice polynomial FUnCtions AND Sugeno integrals}

2.1. Preliminaries. Throughout this paper, let $L$ be an arbitrary bounded chain endowed with lattice operations $\wedge$ and $\vee$, and with least and greatest elements $0_{L}$ and $1_{L}$, respectively; the subscripts may be omitted when the underlying lattice is clear from the context. A subset $S$ of a chain $L$ is said to be convex if for every $a, b \in S$ and every $c \in L$ such that $a \leq c \leq b$, we have $c \in S$. For any subset $S \subseteq L$, we denote by $\operatorname{cl}(S)$ the convex hull of $S$, that is, the smallest convex subset of $L$ containing $S$. For instance, if $a, b \in L$ such that $a \leq b$, then $\operatorname{cl}(\{a, b\})=[a, b]=\{c \in L: a \leq c \leq b\}$.

For an integer $n \geq 1$, we set $[n]=\{1, \ldots, n\}$. Let $\sigma$ be a permutation on $[n]$. The standard simplex of $L^{n}$ associated with $\sigma$ is the subset $L_{\sigma}^{n} \subseteq L^{n}$ defined by

$$
L_{\sigma}^{n}=\left\{\mathbf{x} \in L^{n}: x_{\sigma(1)} \leq x_{\sigma(2)} \leq \cdots \leq x_{\sigma(n)}\right\} .
$$

Two tuples are said to be comonotonic, if there is a standard simplex containing both of them. 
Given arbitrary bounded chains $L_{i}, i \in[n]$, their Cartesian product $\prod_{i \in[n]} L_{i}$ constitutes a bounded distributive lattice by defining

$$
\mathbf{a} \wedge \mathbf{b}=\left(a_{1} \wedge b_{1}, \ldots, a_{n} \wedge b_{n}\right), \quad \text { and } \quad \mathbf{a} \vee \mathbf{b}=\left(a_{1} \vee b_{1}, \ldots, a_{n} \vee b_{n}\right) \text {. }
$$

For $k \in[n]$ and $c \in L_{k}$, we use $\mathbf{x}_{k}^{c}$ to denote the tuple whose $i$ th component is $c$, if $i=k$, and $x_{i}$, otherwise.

For $c \in L$ and $\mathbf{x} \in L^{n}$, let $\mathbf{x} \wedge c=\left(x_{1} \wedge c, \ldots, x_{n} \wedge c\right)$ and $\mathbf{x} \vee c=\left(x_{1} \vee c, \ldots, x_{n} \vee c\right)$, and denote by $[\mathbf{x}]_{c}$ the $n$-tuple whose $i$ th component is 0 , if $x_{i} \leq c$, and $x_{i}$, otherwise, and by $[\mathbf{x}]^{c}$ the $n$-tuple whose $i$ th component is 1 , if $x_{i} \geq c$, and $x_{i}$, otherwise.

Let $f: \prod_{i \in[n]} L_{i} \rightarrow L$ be a function. The range of $f$ is given by $\operatorname{ran}(f)=\{f(\mathbf{x})$ : $\left.\mathbf{x} \in \prod_{i \in[n]} L_{i}\right\}$. Also, $f$ is said to be order-preserving if, for every $\mathbf{a}, \mathbf{b} \in \prod_{i \in[n]} L_{i}$ such that $\mathbf{a} \leq \mathbf{b}$, we have $f(\mathbf{a}) \leq f(\mathbf{b})$. A well-known example of an order-preserving function is the median function med: $L^{3} \rightarrow L$ given by

$$
\operatorname{med}\left(x_{1}, x_{2}, x_{3}\right)=\left(x_{1} \wedge x_{2}\right) \vee\left(x_{1} \wedge x_{3}\right) \vee\left(x_{2} \wedge x_{3}\right) .
$$

Given a tuple $\mathbf{x} \in L^{m}, m \geq 1$, set $\langle\mathbf{x}\rangle_{f}=\operatorname{med}(f(\mathbf{0}), \mathbf{x}, f(\mathbf{1}))$.

2.2. Basic background on polynomial functions and Sugeno integrals. In this subsection we recall some well-known results concerning polynomial functions that will be needed hereinafter. For further background, we refer the reader to [4, 5, 6, 7, 13, 14, 20.

Recall that a (lattice) polynomial function on $L$ is any map $p: L^{n} \rightarrow L$ which can be obtained as a composition of the lattice operations $\wedge$ and $\vee$, the projections $\mathbf{x} \mapsto x_{i}$ and the constant functions $\mathbf{x} \mapsto c, c \in L$.

Polynomial functions are known to generalize certain prominent fuzzy integrals, namely, the so-called (discrete) Sugeno integrals. Indeed, as observed in [18, 19, Sugeno integrals coincide exactly with those polynomial functions $q: L^{n} \rightarrow L$ which are idempotent, that is, which satisfy $q(c, \ldots, c)=c$, for every $c \in L$. In particular we have $\operatorname{ran}(q)=L$. We shall take this as our working definition of the Sugeno integral; for the original definition (as an integral with respect to a fuzzy measure) see, e.g. [16, 21, 22].

As shown by Goodstein [13, polynomial functions over bounded distributive lattices (in particular, over bounded chains) have very neat normal form representations. For $I \subseteq[n]$, let $\mathbf{e}_{I}$ be the characteristic vector of $I$, i.e., the $n$-tuple in $L^{n}$ whose $i$ th component is 1 if $i \in I$, and 0 otherwise.

Theorem 1. A function $p: L^{n} \rightarrow L$ is a polynomial function if and only if

$$
p\left(x_{1}, \ldots, x_{n}\right)=\bigvee_{I \subseteq[n]}\left(p\left(\mathbf{e}_{I}\right) \wedge \bigwedge_{i \in I} x_{i}\right) .
$$

Furthermore, the function given by (2) is a Sugeno integral if and only if $p(\mathbf{0})=0$ and $p(\mathbf{1})=1$.

Remark 1. Observe that, by Theorem 1 every polynomial function $p: L^{n} \rightarrow L$ is uniquely determined by its restriction to $\{0,1\}^{n}$. Also, since every lattice polynomial function is order-preserving, we have that the coefficients in (2) are monotone increasing, i.e., $p\left(\mathbf{e}_{I}\right) \leq p\left(\mathbf{e}_{J}\right)$ whenever $I \subseteq J$. Moreover, a function $f:\{0,1\}^{n} \rightarrow L$ can be extended to a polynomial function over $L$ if and only if it is order-preserving.

Remark 2. It follows from Goodstein's theorem that every unary polynomial function is of the form

$$
p(x)=s \vee(x \wedge t)=\operatorname{med}(s, x, t)= \begin{cases}s, & \text { if } x<s, \\ x, & \text { if } x \in[s, t], \\ t, & \text { if } t<x,\end{cases}
$$




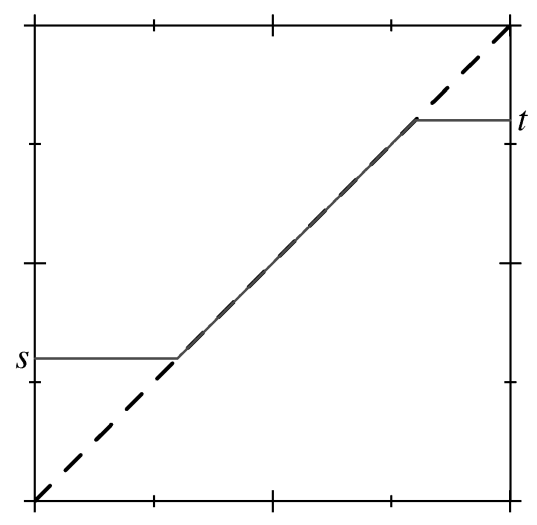

Figure 1. A typical unary polynomial function

where $s=p(0), t=p(1)$. In other words, $p(x)$ is a truncated identity function. Figure 1 shows the graph of this function in the case when $L$ is the real unit interval $[0,1]$.

It is noteworthy that every polynomial function $p$ as in 2 can be represented by $p=\langle q\rangle_{p}$ where $q$ is the Sugeno integral given by

$$
q\left(x_{1}, \ldots, x_{n}\right)=\bigvee_{\emptyset \subsetneq I \subsetneq[n]}\left(p\left(\mathbf{e}_{I}\right) \wedge \bigwedge_{i \in I} x_{i}\right) \vee \bigwedge_{i \in[n]} x_{i}
$$

2.3. Characterizations of polynomial functions. The following results reassemble the various characterizations of polynomial functions obtained in [5]. For further background see, e.g. [6, 7, 16].

Theorem 2. Let $p: L^{n} \rightarrow L$ be a function on an arbitrary bounded chain $L$. The following conditions are equivalent:

(i) $p$ is a polynomial function.

(ii) $p$ is median decomposable, that is, for every $\mathbf{x} \in L^{n}$,

$$
p(\mathbf{x})=\operatorname{med}\left(p\left(\mathbf{x}_{k}^{0}\right), x_{k}, p\left(\mathbf{x}_{k}^{1}\right)\right) \quad(k=1, \ldots, n) .
$$

(iii) $p$ is order-preserving, and $\mathrm{cl}(\operatorname{ran}(p))$-min and $\mathrm{cl}(\operatorname{ran}(p))$-max homogeneous, that is, for every $\mathrm{x} \in L^{n}$ and every $c \in \operatorname{cl}(\operatorname{ran}(p))$,

$$
p(\mathbf{x} \wedge c)=p(\mathbf{x}) \wedge c \quad \text { and } \quad p(\mathbf{x} \vee c)=p(\mathbf{x}) \vee c, \quad \text { resp } .
$$

(iv) $p$ is order-preserving, range-idempotent, and horizontally minitive and maxitive, that is, for every $\mathbf{x} \in L^{n}$ and every $c \in L$,

$$
p(\mathbf{x})=p(\mathbf{x} \vee c) \wedge p\left([\mathbf{x}]^{c}\right) \text { and } p(\mathbf{x})=p(\mathbf{x} \wedge c) \vee p\left([\mathbf{x}]_{c}\right), \quad \text { resp. }
$$

Remark 3. Note that, by the equivalence (i) $\Leftrightarrow$ (iii), for every polynomial function $p: L^{n} \rightarrow L, p(\mathbf{x})=\langle p(\mathbf{x})\rangle_{p}=p\left(\langle\mathbf{x}\rangle_{p}\right)$. Moreover, for every function $f: L^{m} \rightarrow L$ and every Sugeno integral $q: L^{n} \rightarrow L$, we have $\langle q(\mathbf{x})\rangle_{f}=q\left(\langle\mathbf{x}\rangle_{f}\right)$.

Remark 4. The concepts of $S$-min and $S$-max homogeneity were used by Fodor and Roubens [12] to axiomatize certain classes of aggregation functions in the case when $S=L$ is the real interval $[0,1]$. The concept of horizontal maxitivity was introduced by Benvenuti et al. 22, also in the case when $L$ is the real interval [0,1], as a general property of the Sugeno integral. 
Theorem 2 is a refinement of the Main Theorem in [5] originally stated for functions over bounded distributive lattices. As shown in [7, in the case when $L$ is a chain, Theorem 2 can be strengthened since the conditions need to be verified only on tuples of certain prescribed types. Moreover, further characterizations are available and given in terms of conditions of somewhat different flavor, as the following theorem illustrates [7.

Theorem 3. A function $p: L^{n} \rightarrow L$ is a polynomial function if and only if it is range-idempotent, and comonotonic minitive and maxitive, that is, for any two comonotonic tuples $\mathbf{x}$ and $\mathbf{x}^{\prime}$ we have

$$
p\left(\mathbf{x} \wedge \mathbf{x}^{\prime}\right)=p(\mathbf{x}) \wedge p\left(\mathbf{x}^{\prime}\right) \quad \text { and } \quad p\left(\mathbf{x} \vee \mathbf{x}^{\prime}\right)=p(\mathbf{x}) \vee p\left(\mathbf{x}^{\prime}\right), \quad \text { respectively } .
$$

\section{Pseudo-Sugeno integrals and Sugeno utility functions}

In this section we study certain prominent function classes in the realm of multicriteria decision making. More precisely, we investigate overall utility functions $U: \prod_{i \in[n]} L_{i} \rightarrow L$ which can be obtained by aggregating various local utility functions (i.e., order-preserving mappings) $\varphi_{i}: L_{i} \rightarrow L, i \in[n]$, using Sugeno integrals.

To this extent, in Subsection 3.1 we introduce the wider class of pseudo-polynomial functions, and we present their axiomatization in Subsection 3.2. As we will see, pseudo-polynomial functions can be equivalently defined in terms of Sugeno integrals, and thus they model certain processes within multicriteria decision making. This is observed in Subsection 3.3 where the notion of a Sugeno utility function $U: \prod_{i \in[n]} L_{i} \rightarrow L$ associated with given local utility functions $\varphi_{i}: L_{i} \rightarrow L, i \in[n]$, is discussed. Using the axiomatization of pseudo-polynomial functions, in Subsection 3.4 we establish several characterizations of Sugeno utility functions given in terms of necessary and sufficient conditions which naturally extend those presented in Subsection 2.3.

3.1. Pseudo-Sugeno integrals and pseudo-polynomial functions. Let $L$ and $L_{1}, \ldots, L_{n}$ be bounded chains. We shall denote the top and bottom elements of $L_{1}, \ldots, L_{n}$ and $L$ by 1 and 0 , respectively. This convention will not give rise to ambiguities. We shall say that a mapping $\varphi_{i}: L_{i} \rightarrow L, i \in[n]$, satisfies the boundary conditions if for every $x \in L_{i}$,

$$
\varphi_{i}(0) \leq \varphi_{i}(x) \leq \varphi_{i}(1) \quad \text { or } \quad \varphi_{i}(1) \leq \varphi_{i}(x) \leq \varphi_{i}(0) .
$$

Observe that if $\varphi_{i}$ is order-preserving, then it satisfies the boundary conditions. To simplify our exposition, we will assume that $\varphi_{i}(0) \leq \varphi_{i}(x) \leq \varphi_{i}(1)$ holds; this can be always achieved by replacing $L_{i}$ by its dual if necessary.

A function $f: \prod_{i \in[n]} L_{i} \rightarrow L$ is a pseudo-polynomial function if there is a polynomial function $p: L^{n} \rightarrow L$ and there are unary functions $\varphi_{i}: L_{i} \rightarrow L, i \in[n]$, satisfying the boundary conditions, such that

$$
f(\mathbf{x})=p\left(\varphi_{1}\left(x_{1}\right), \ldots, \varphi_{n}\left(x_{n}\right)\right) .
$$

If $p$ is a Sugeno integral, then we say that $f$ is a pseudo-Sugeno integral. As the following result asserts, the notions of pseudo-polynomial function and pseudo-Sugeno integral turn out to be equivalent.

Proposition 1. A function $f: \prod_{i \in[n]} L_{i} \rightarrow L$ is a pseudo-polynomial function if and only if it is a pseudo-Sugeno integral.

Proof. Clearly, every pseudo-Sugeno integral is a pseudo-polynomial function. Conversely, if $f: \prod_{i \in[n]} L_{i} \rightarrow L$ is a function of the form $f(\mathbf{x})=p\left(\varphi_{1}\left(x_{1}\right), \ldots, \varphi_{n}\left(x_{n}\right)\right)$ 
for a lattice polynomial $p$, then by setting $\phi_{i}=\left\langle\varphi_{i}\right\rangle_{p}$ and taking $q$ as a Sugeno integral such that $p=\langle q\rangle_{p}$, we have

$$
\begin{aligned}
f(\mathbf{x}) & =\left\langle q\left(\varphi_{1}\left(x_{1}\right), \ldots, \varphi_{n}\left(x_{n}\right)\right)\right\rangle_{p}=q\left(\left\langle\varphi_{1}\left(x_{1}\right)\right\rangle_{p}, \ldots,\left\langle\varphi_{n}\left(x_{n}\right)\right\rangle_{p}\right) \\
& =q\left(\phi_{1}\left(x_{1}\right), \ldots, \phi_{n}\left(x_{n}\right)\right),
\end{aligned}
$$

and thus $f$ is a pseudo-Sugeno integral.

Remark 5. Clearly, if $f(\mathbf{x})=p\left(\varphi_{1}\left(x_{1}\right), \ldots, \varphi_{n}\left(x_{n}\right)\right)$ is a pseudo-polynomial function, then for all $k \in[n]$ and $\mathbf{x} \in \prod_{i \in[n]} L_{i}$ we have

$$
f\left(\mathbf{x}_{k}^{0}\right) \leq f(\mathbf{x}) \leq f\left(\mathbf{x}_{k}^{1}\right) .
$$

3.2. A characterization of pseudo-Sugeno integrals. Throughout this subsection, we assume that the unary maps $\varphi_{i}: L_{i} \rightarrow L$ considered, satisfy the boundary condition $\varphi_{i}(0) \leq \varphi_{i}(x) \leq \varphi_{i}(1)$.

We say that $f: \prod_{i \in[n]} L_{i} \rightarrow L$ is pseudo-median decomposable if for each $k \in[n]$ there is a unary function $\varphi_{k}: L_{k} \rightarrow L$ such that

$$
f(\mathbf{x})=\operatorname{med}\left(f\left(\mathbf{x}_{k}^{0}\right), \varphi_{k}\left(x_{k}\right), f\left(\mathbf{x}_{k}^{1}\right)\right)
$$

for every $\mathbf{x} \in \prod_{i \in[n]} L_{i}$. Note that if $f$ is pseudo-median decomposable w.r.t. unary functions $\varphi_{i}: L_{i} \rightarrow L, i \in[n]$, then (5) holds.

Theorem 4. Let $f: \prod_{i \in[n]} L_{i} \rightarrow L$ be a function. Then $f$ is a pseudo-Sugeno integral if and only if $f$ is pseudo-median decomposable.

Proof. First we show that the condition is necessary. Suppose that the function $f: \prod_{i \in[n]} L_{i} \rightarrow L$ is of the form $f(\mathbf{x})=q\left(\varphi_{1}\left(x_{1}\right), \ldots, \varphi_{n}\left(x_{n}\right)\right)$ for some Sugeno integral $q$ and unary functions $\varphi_{k}$ satisfying the boundary conditions. We prove (6) for $k=1$; the other cases can be dealt with similarly. Let us fix the values of $x_{2}, \ldots, x_{n}$, and let us consider the unary polynomial function $u(y)=q\left(y, \varphi_{2}\left(x_{2}\right), \ldots, \varphi_{n}\left(x_{n}\right)\right)$.

Setting $a=\varphi_{1}(0), b=\varphi_{1}(1), y_{1}=\varphi_{1}\left(x_{1}\right)$, the equality to prove takes the form $u\left(y_{1}\right)=\operatorname{med}\left(u(a), y_{1}, u(b)\right)$. This becomes clear if we take into account that $u$ is of the form (3), and by the boundary condition $a \leq y_{1} \leq b$ (see also Figure 1).

To verify that the condition is sufficient, just observe that applying (6) repeatedly to each variable of $f$ we can straightforwardly obtain a representation of $f$ as $f(\mathbf{x})=p\left(\varphi_{1}\left(x_{1}\right), \ldots, \varphi_{n}\left(x_{n}\right)\right)$ for some polynomial function $p$. Thus, $f$ is a pseudopolynomial function and, by Proposition 1 it is a pseudo-Sugeno integral.

In the next theorem we give a disjunctive normal form of the polynomial $p$ obtained at the end of the proof of the above theorem (by repeated applications of the pseudomedian decomposition formula). Here $\mathbf{e}_{I}$ denotes the characteristic vector of $I \subseteq[n]$ in $\prod_{i \in[n]} L_{i}$, i.e., the $n$-tuple in $\prod_{i \in[n]} L_{i}$ whose $i$-th component is $1_{L_{i}}$ if $i \in I$, and $0_{L_{i}}$ otherwise.

Theorem 5. If $f: \prod_{i \in[n]} L_{i} \rightarrow L$ is pseudo-median decomposable w.r.t. unary functions $\varphi_{i}: L_{i} \rightarrow L, i \in[n]$, then $f(\mathbf{x})=p\left(\varphi_{1}\left(x_{1}\right), \ldots, \varphi_{n}\left(x_{n}\right)\right)$, where $p$ is given by

$$
p\left(x_{1}, \ldots, x_{n}\right)=\bigvee_{I \subseteq[n]}\left(f\left(\mathbf{e}_{I}\right) \wedge \bigwedge_{i \in I} x_{i}\right) .
$$

Proof. We need to prove that the following identity holds:

$$
f\left(x_{1}, \ldots, x_{n}\right)=\bigvee_{I \subseteq[n]}\left(f\left(\mathbf{e}_{I}\right) \wedge \bigwedge_{i \in I} \varphi_{i}\left(x_{i}\right)\right) .
$$

We proceed by induction on $n$. If $n=1$, then the right hand side of (7) takes the form $f(0) \vee\left(f(1) \wedge \varphi_{1}\left(x_{1}\right)\right)=\operatorname{med}\left(f(0), \varphi_{1}\left(x_{1}\right), f(1)\right)$, which equals $f\left(x_{1}\right)$ 
by (6). Now suppose that the statement of the theorem is true for all pseudomedian decomposable functions in $n-1$ variables. Applying the pseudo-median decomposition to $f$ with $k=n$ we obtain

$$
\begin{aligned}
f\left(x_{1}, \ldots, x_{n}\right) & =\operatorname{med}\left(f_{0}\left(x_{1}, \ldots, x_{n-1}\right), \varphi_{n}\left(x_{n}\right), f_{1}\left(x_{1}, \ldots, x_{n-1}\right)\right) \\
& =f_{0}\left(x_{1}, \ldots, x_{n-1}\right) \vee\left(f_{1}\left(x_{1}, \ldots, x_{n-1}\right) \wedge \varphi_{n}\left(x_{n}\right)\right),
\end{aligned}
$$

where $f_{0}$ and $f_{1}$ are the $(n-1)$-ary functions defined by

$$
\begin{aligned}
& f_{0}\left(x_{1}, \ldots, x_{n-1}\right)=f\left(x_{1}, \ldots, x_{n-1}, 0\right), \\
& f_{1}\left(x_{1}, \ldots, x_{n-1}\right)=f\left(x_{1}, \ldots, x_{n-1}, 1\right) .
\end{aligned}
$$

It is easy to verify that $f_{0}$ and $f_{1}$ are pseudo-median decomposable w.r.t. $\varphi_{1}, \ldots, \varphi_{n-1}$, therefore we can apply the induction hypothesis to these functions:

$$
\begin{aligned}
f_{0}\left(x_{1}, \ldots, x_{n-1}\right)= & \bigvee_{I \subseteq[n-1]}\left(f_{0}\left(\mathbf{e}_{I}\right) \wedge \bigwedge_{i \in I} \varphi_{i}\left(x_{i}\right)\right)= \\
& \bigvee_{I \subseteq[n-1]}\left(f\left(\mathbf{e}_{I}\right) \wedge \bigwedge_{i \in I} \varphi_{i}\left(x_{i}\right)\right), \\
f_{1}\left(x_{1}, \ldots, x_{n-1}\right)= & \bigvee_{I \subseteq[n-1]}\left(f_{1}\left(\mathbf{e}_{I}\right) \wedge \bigwedge_{i \in I} \varphi_{i}\left(x_{i}\right)\right)= \\
& \bigvee_{I \subseteq[n-1]}\left(f\left(\mathbf{e}_{I \cup\{n\}}\right) \wedge \bigwedge_{i \in I} \varphi_{i}\left(x_{i}\right)\right) .
\end{aligned}
$$

Substituting back into (8) and using distributivity we obtain the desired equality (7).

Let us note that the polynomial $p$ given in the above theorem is a Sugeno integral if and only if $f(\mathbf{0})=0$ and $f(\mathbf{1})=1$.

3.3. Motivation: overall utility functions. Despite the theoretical interest, the motivation for the study of pseudo-Sugeno integrals (or, equivalently, pseudo-polynomial functions) is deeply rooted in multicriteria decision making. Let $\varphi_{i}: L_{i} \rightarrow L$, $i \in[n]$, be local utility functions (i.e., order-preserving mappings) having a common range $\mathcal{R} \subseteq L$, and let $M: L^{n} \rightarrow L$ be an aggregation function. The overall utility function associated with $\varphi_{i}, i \in[n]$, and $M$ is the mapping $U: \prod_{i \in[n]} L_{i} \rightarrow L$ defined by

$$
U(\mathbf{x})=M\left(\varphi_{1}\left(x_{1}\right), \ldots, \varphi_{n}\left(x_{n}\right)\right) .
$$

For background on overall utility functions, see e.g. [3, 15].

Thus, pseudo-Sugeno integrals subsume those overall utility functions (9) where the aggregation function $M$ is a Sugeno integral. In the sequel we shall refer to a mapping $f: \prod_{i \in[n]} L_{i} \rightarrow L$ for which there are local utility functions $\varphi_{i}, i \in[n]$, and a Sugeno integral (or, equivalently, a polynomial function) $q$, such that

$$
f(\mathbf{x})=q\left(\varphi_{1}\left(x_{1}\right), \ldots, \varphi_{n}\left(x_{n}\right)\right),
$$

as a Sugeno utility function. As it will become clear in Corollary 2, these Sugeno utility functions coincide exactly with those pseudo-Sugeno integrals (or equivalently, pseudo-polynomial functions) which are order-preserving. Also, by taking $L_{1}=\cdots=$ $L_{n}=L$ and $\varphi_{1}=\cdots=\varphi_{n}=\varphi$, it follows that Sugeno utility functions subsume the notions of quasi-Sugeno integral and quasi-polynomial function in the terminology of 8 .

Remark 6. Note that the condition that $\varphi_{i}: L_{i} \rightarrow L, i \in[n]$ have a common range $\mathcal{R}$ is not really restrictive, since each $\varphi_{i}$ can be extended to a local utility function $\varphi_{i}^{\prime}: L_{i}^{\prime} \rightarrow L$, where $L_{i} \subseteq L_{i}^{\prime}$, in such a way that each $\varphi_{i}^{\prime}, i \in[n]$, has the same range 
$\mathcal{R} \subseteq L$. In fact, if $\mathcal{R}_{i}$ is the range of $\varphi_{i}$, for each $i \in[n]$, then $\mathcal{R}$ can be chosen as the interval

$$
\operatorname{cl}\left(\bigcup_{i \in[n]} \mathcal{R}_{i}\right)=\left[\bigwedge_{i \in[n]} \varphi_{i}(0), \bigvee_{i \in[n]} \varphi_{i}(1)\right]
$$

In this way, if $f^{\prime}: \prod_{i \in[n]} L_{i}^{\prime} \rightarrow L$ is such that $f^{\prime}(\mathbf{x})=q\left(\varphi_{1}^{\prime}\left(x_{1}\right), \ldots, \varphi_{n}^{\prime}\left(x_{n}\right)\right)$, then the restriction of $f^{\prime}$ to $\prod_{i \in[n]} L_{i}$ coincides with the function $f(\mathbf{x})=q\left(\varphi_{1}\left(x_{1}\right), \ldots, \varphi_{n}\left(x_{n}\right)\right)$.

3.4. Characterizations of Sugeno utility functions. In view of the remark above, in this subsection we will assume that the local utility functions $\varphi_{i}: L_{i} \rightarrow L$, $i \in[n]$, considered have the same range $\mathcal{R} \subseteq L$. Since local utility functions satisfy the boundary conditions, from Theorem 4 we get the following characterization of Sugeno utility functions.

Corollary 1. A function $f: \prod_{i \in[n]} L_{i} \rightarrow L$ is a Sugeno utility function if and only if it is pseudo-median decomposable w.r.t. local utility functions.

We will provide further axiomatizations of Sugeno utility functions extending those of polynomial functions given in Subsection 2.3 as well as those of quasipolynomial functions given in 8]. For the sake of simplicity, given $\varphi_{i}: L_{i} \rightarrow L$, $i \in[n]$, we make use of the shorthand notation $\bar{\varphi}(\mathbf{x})=\left(\varphi_{1}\left(x_{1}\right), \ldots, \varphi_{n}\left(x_{n}\right)\right)$ and $\bar{\varphi}^{-1}(c)=\left\{\mathbf{d}: \varphi_{i}\left(d_{i}\right)=c\right.$ for all $\left.i \in[n]\right\}$, for every $c \in \mathcal{R}$.

We say that a function $f: \prod_{i \in[n]} L_{i} \rightarrow L$ is pseudo-max homogeneous (resp. pseudo-min homogeneous) if there are local utility functions $\varphi_{i}: L_{i} \rightarrow L, i \in[n]$, such that for every $\mathbf{x} \in \prod_{i \in[n]} L_{i}$ and $c \in \mathcal{R}$,

$$
f(\mathbf{x} \vee \mathbf{d})=f(\mathbf{x}) \vee c(\text { resp. } f(\mathbf{x} \wedge \mathbf{d})=f(\mathbf{x}) \wedge c) \text {, whenever } \mathbf{d} \in \bar{\varphi}^{-1}(c) .
$$

Fact 1. Let $f: \prod_{i \in[n]} L_{i} \rightarrow L$ be a function, and let $\varphi_{i}: L_{i} \rightarrow L, i \in[n]$, be local utility functions. If $f$ is pseudo-min homogeneous and pseudo-max homogeneous w.r.t. $\varphi_{1}, \ldots, \varphi_{n}$, then it satisfies the condition

$$
\text { for every } c \in \mathcal{R} \text { and } \mathbf{d} \in \bar{\varphi}^{-1}(c), f(\mathbf{d})=c \text {. }
$$

Lemma 1. If $f\left(x_{1}, \ldots, x_{n}\right)=q\left(\varphi\left(x_{1}\right), \ldots, \varphi_{n}\left(x_{n}\right)\right)$ for some Sugeno integral $q: L^{n} \rightarrow$ $L$ and local utility functions $\varphi_{1}, \ldots, \varphi_{n}$, then $f$ is pseudo-min homogeneous and pseudo-max homogeneous w.r.t. $\varphi_{1}, \ldots, \varphi_{n}$.

Proof. Let $\mathcal{R}$ be the common range of $\varphi_{1}, \ldots, \varphi_{n}$, let $c \in \mathcal{R}$ and $\mathbf{d} \in \bar{\varphi}^{-1}(c)$. By Theorem 2 and the fact that each $\varphi_{k}$ is order-preserving, we have

$$
\begin{aligned}
f(\mathbf{x} \vee \mathbf{d}) & =q(\bar{\varphi}(\mathbf{x} \vee \mathbf{d}))=q(\bar{\varphi}(\mathbf{x}) \vee \bar{\varphi}(\mathbf{d})) \\
& =q(\bar{\varphi}(\mathbf{x}) \vee c)=q(\bar{\varphi}(\mathbf{x})) \vee c=f(\mathbf{x}) \vee c,
\end{aligned}
$$

and hence, $f$ is pseudo-max homogeneous. The dual statement follows similarly.

For $\mathbf{x}, \mathbf{d} \in \prod_{i \in[n]} L_{i}$, let $[\mathbf{x}]_{\mathbf{d}}$ be the $n$-tuple whose $i$ th component is $0_{L_{i}}$, if $x_{i} \leq d_{i}$, and $x_{i}$, otherwise, and dually let $[\mathbf{x}]^{\mathbf{d}}$ be the $n$-tuple whose $i$ th component is $1_{L_{i}}$, if $x_{i} \geq d_{i}$, and $x_{i}$, otherwise. We say that $f: \prod_{i \in[n]} L_{i} \rightarrow L$ is pseudo-horizontally maxitive (resp. pseudo-horizontally minitive) if there are local utility functions $\varphi_{i}: L_{i} \rightarrow L, i \in[n]$, such that for every $\mathbf{x} \in \prod_{i \in[n]} L_{i}$ and $c \in \mathcal{R}$, if $\mathbf{d} \in \bar{\varphi}^{-1}(c)$, then

$$
f(\mathbf{x})=f(\mathbf{x} \wedge \mathbf{d}) \vee f\left([\mathbf{x}]_{\mathbf{d}}\right) \quad\left(\text { resp. } f(\mathbf{x})=f(\mathbf{x} \vee \mathbf{d}) \wedge f\left([\mathbf{x}]^{\mathbf{d}}\right)\right) .
$$

Lemma 2. If $f: \prod_{i \in[n]} L_{i} \rightarrow L$ is order-preserving, pseudo-horizontally minitive (resp. pseudo-horizontally maxitive) and satisfies (12), then it is pseudo-min homogeneous (resp. pseudo-max homogeneous). 
Proof. If $f: \prod_{i \in[n]} L_{i} \rightarrow L$ is order-preserving, pseudo-horizontally minitive and satisfies 12 w.r.t. $\varphi_{1}, \ldots, \varphi_{n}$, then for every $\mathbf{x} \in \prod_{i \in[n]} L_{i}, c \in \mathcal{R}, \mathbf{d} \in \bar{\varphi}^{-1}(c)$ we have

$$
\begin{aligned}
f(\mathbf{x}) \wedge c & =f(\mathbf{x}) \wedge f(\mathbf{d}) \geq f(\mathbf{x} \wedge \mathbf{d})=f((\mathbf{x} \wedge \mathbf{d}) \vee \mathbf{d}) \wedge f\left([\mathbf{x} \wedge \mathbf{d}]^{\mathbf{d}}\right) \\
& =f(\mathbf{d}) \wedge f\left([\mathbf{x}]^{\mathbf{d}}\right) \geq f(\mathbf{d}) \wedge f(\mathbf{x})=f(\mathbf{x}) \wedge c .
\end{aligned}
$$

Hence $f$ is pseudo-min homogeneous w.r.t. $\varphi_{1}, \ldots, \varphi_{n}$. The dual statement can be proved similarly.

Lemma 3. Suppose that $f: \prod_{i \in[n]} L_{i} \rightarrow L$ is order-preserving and pseudo-min homogeneous (resp. pseudo-max homogeneous), and satisfies (12). Then $f$ is pseudomax homogeneous (resp. pseudo-min homogeneous) if and only if it is pseudohorizontally maxitive (resp. pseudo-horizontally minitive).

Proof. Suppose that $f: \prod_{i \in[n]} L_{i} \rightarrow L$ is order-preserving and pseudo-min homogeneous and satisfies (12) w.r.t. $\varphi_{1}, \ldots, \varphi_{n}$. Assume first that $f$ is pseudo-max homogeneous w.r.t. $\varphi_{1}, \ldots, \varphi_{n}$. For every $\mathbf{x} \in \prod_{i \in[n]} L_{i}$ and $\mathbf{d} \in \bar{\varphi}^{-1}(c)$, where $c \in \mathcal{R}$, we have

$$
\begin{aligned}
f(\mathbf{x} \wedge \mathbf{d}) \vee f\left([\mathbf{x}]_{\mathbf{d}}\right) & =(f(\mathbf{x}) \wedge c) \vee f\left([\mathbf{x}]_{\mathbf{d}}\right)=\left(f(\mathbf{x}) \vee f\left([\mathbf{x}]_{\mathbf{d}}\right)\right) \wedge\left(c \vee f\left([\mathbf{x}]_{\mathbf{d}}\right)\right) \\
& =f(\mathbf{x}) \wedge f\left(\mathbf{d} \vee[\mathbf{x}]_{\mathbf{d}}\right)=f(\mathbf{x}),
\end{aligned}
$$

and hence $f$ is pseudo-horizontally maxitive w.r.t. $\varphi_{1}, \ldots, \varphi_{n}$.

Conversely, if $f$ is pseudo-horizontally maxitive w.r.t. $\varphi_{1}, \ldots, \varphi_{n}$, then by Lemma $2 f$ is pseudo-max homogeneous w.r.t. $\varphi_{1}, \ldots, \varphi_{n}$. The dual statement can be proved similarly.

Lemma 4. If $f: \prod_{i \in[n]} L_{i} \rightarrow L$ is order-preserving, pseudo-min homogeneous and pseudo-horizontally maxitive, then it is pseudo-median decomposable w.r.t. local utility functions.

Proof. Let $\mathbf{x} \in \prod_{i \in[n]} L_{i}$ and let $k \in[n]$. If $f$ is pseudo-horizontally maxitive, say w.r.t. $\varphi_{1}, \ldots, \varphi_{n}$, then $f(\mathbf{x})=f(\mathbf{x} \wedge \mathbf{d}) \vee f\left([\mathbf{x}]_{\mathbf{d}}\right)$ holds for every $\mathbf{d} \in \bar{\varphi}^{-1}\left(\varphi_{k}\left(x_{k}\right)\right)$ whose $k$ th component is $x_{k}$. Now if $f$ is pseudo-min homogeneous, then $f(\mathbf{x} \wedge \mathbf{d})=$ $f\left(\mathbf{x}_{k}^{1} \wedge \mathbf{d}\right)=f\left(\mathbf{x}_{k}^{1}\right) \wedge \varphi_{k}\left(x_{k}\right)$, and by the definition of $[\mathbf{x}]_{\mathbf{d}}$, we have $f\left([\mathbf{x}]_{\mathbf{d}}\right) \leq f\left(\mathbf{x}_{k}^{0}\right)$. Thus,

$$
\begin{aligned}
f(\mathbf{x}) & =\operatorname{med}\left(f\left(\mathbf{x}_{k}^{0}\right), f(\mathbf{x}), f\left(\mathbf{x}_{k}^{1}\right)\right)=\left(f\left(\mathbf{x}_{k}^{0}\right) \vee f(\mathbf{x})\right) \wedge f\left(\mathbf{x}_{k}^{1}\right) \\
& =\left(f\left(\mathbf{x}_{k}^{0}\right) \vee\left(f\left(\mathbf{x}_{k}^{1}\right) \wedge \varphi_{k}\left(x_{k}\right)\right) \wedge f\left(\mathbf{x}_{k}^{1}\right)=f\left(\mathbf{x}_{k}^{0}\right) \vee\left(f\left(\mathbf{x}_{k}^{1}\right) \wedge \varphi_{k}\left(x_{k}\right)\right)\right. \\
& =\operatorname{med}\left(f\left(\mathbf{x}_{k}^{0}\right), \varphi_{k}\left(x_{k}\right), f\left(\mathbf{x}_{k}^{1}\right)\right) .
\end{aligned}
$$

Since this holds for every $\mathbf{x} \in \prod_{i \in[n]} L_{i}$ and $k \in[n], f$ is pseudo-median decomposable.

We can also extend the comonotonic properties as follows. We say that a function $f: \prod_{i \in[n]} L_{i} \rightarrow L$ is pseudo-comonotonic minitive (resp. pseudo-comonotonic maxitive) if there are local utility functions $\varphi_{i}: L_{i} \rightarrow L, i \in[n]$, such that for every $\mathbf{x}$ and $\mathbf{x}^{\prime}$, if $\bar{\varphi}(\mathbf{x})$ and $\bar{\varphi}\left(\mathbf{x}^{\prime}\right)$ are comonotonic, then

$$
f\left(\mathbf{x} \wedge \mathbf{x}^{\prime}\right)=f(\mathbf{x}) \wedge f\left(\mathbf{x}^{\prime}\right) \quad\left(\text { resp. } f\left(\mathbf{x} \vee \mathbf{x}^{\prime}\right)=f(\mathbf{x}) \vee f\left(\mathbf{x}^{\prime}\right)\right) .
$$

The following fact is straightforward.

Fact 2. Every Sugeno utility function of the form 10 is pseudo-comonotonic minitive and maxitive. Moreover, if a function is pseudo-comonotonic minitive (resp. pseudo-comonotonic maxitive) and satisfies (12), then it is pseudo-min homogeneous (resp. pseudo-max homogeneous). 
Let $\mathbf{P}$ be the set comprising the properties of pseudo-min homogeneity, pseudohorizontal minitivity and pseudo-comonotic minitivity, and let $\mathbf{P}^{d}$ be the set comprising the corresponding dual properties. The following result generalizes the various characterizations of polynomial functions given in Subsection 2.3

Theorem 6. Let $f: \prod_{i \in[n]} L_{i} \rightarrow L$ be an order-preserving function. The following assertions are equivalent:

(i) $f$ is a Sugeno utility function.

(ii) $f$ is pseudo-median decomposable w.r.t. local utility functions.

(iii) $f$ is $P_{1} \in \mathbf{P}$ and $P_{2} \in \mathbf{P}^{d}$, and satisfies 12 .

Proof. By Corollary 1, we have (i) $\Leftrightarrow$ (ii). By Lemma 1 we also have that if (i) holds, then $f$ is pseudo-min homogeneous and pseudo-max homogeneous. Furthermore, by Fact 2 and Lemmas 2, 3 and 4, we have that any two formulations of (iii) are equivalent. By Lemma 4 (iii) $\Rightarrow$ (ii).

Remark 7. By Fact 1, if $P_{1}$ and $P_{2}$ are the pseudo-homogeneity properties, then (12) becomes redundant in (iii). Similarly, by Lemma 4. Corollary 1, and (i) $\Rightarrow$ (iii) of Theorem 6 if $P_{1}$ is pseudo-min homogeneity (pseudo-horizontal minitivity) property, and $P_{2}$ is pseudo-horizontal maxitivity (pseudo-max homogeneity) property, then (12) is redundant in (iii).

Remark 8. Note that if a function is pseudo-comonotonic minitive or pseudocomonotonic maxitive (w.r.t. $\varphi_{k}: L_{k} \rightarrow L, k \in[n]$ ), then it is order-preserving on every set

$$
S_{\varphi, \sigma}^{n}=\left\{\mathbf{x} \in \prod_{i \in[n]} L_{i}: \bar{\varphi}(\mathbf{x}) \in L_{\sigma}^{n}\right\} \subseteq \prod_{i \in[n]} L_{i} .
$$

As it turns out, this fact can be extended to the whole domain $\prod_{i \in[n]} L_{i}$. To illustrate, let $\mathbf{x}=\left(x_{1}, x_{2}, \ldots, x_{n}\right) \in L^{n}$ and $\mathbf{y}=\left(y_{1}, x_{2}, \ldots, x_{n}\right) \in L^{n}$ such that $\bar{\varphi}(\mathbf{x})$ and $\bar{\varphi}(\mathbf{y})$ are not comonotonic, say

$$
\begin{aligned}
& \varphi_{1}\left(x_{1}\right)<\varphi_{2}\left(x_{2}\right) \leq \varphi_{3}\left(x_{3}\right) \leq \cdots \leq \varphi_{n}\left(x_{n}\right), \\
& \varphi_{2}\left(x_{2}\right)<\varphi_{1}\left(y_{1}\right) \leq \varphi_{3}\left(x_{3}\right) \leq \cdots \leq \varphi_{n}\left(x_{n}\right) .
\end{aligned}
$$

Since $\varphi_{1}$ and $\varphi_{2}$ have the same range, there exists $z_{1} \in L_{1}$ such that $\varphi_{1}\left(z_{1}\right)=\varphi_{2}\left(x_{2}\right)$. Then, for $\mathbf{z}=\left(z_{1}, x_{2}, \ldots, x_{n}\right), \bar{\varphi}(\mathbf{z})$ is comonotonic with $\bar{\varphi}(\mathbf{x})$ and $\bar{\varphi}(\mathbf{y})$, and $\mathbf{x}<\mathbf{z}<$ y. Now, if $f: \prod_{i \in[n]} L_{i} \rightarrow L$ is pseudo-comonotonic minitive or pseudo-comonotonic maxitive (w.r.t. $\left.\varphi_{k}: L_{k} \rightarrow L, k=1, \ldots, n\right)$, then $f(\mathbf{x}) \leq f(\mathbf{z}) \leq f(\mathbf{y})$. The same idea, taking middle-points and applying it componentwise, can be used to show that if a function is pseudo-comonotonic minitive or pseudo-comonotonic maxitive, then it is order-preserving.

\section{Factorization of Sugeno Utility functions}

In this section we present an algorithm that decides whether a given function $f: \prod_{i \in[n]} L_{i} \rightarrow L$ has a factorization of the form $10 p$ and constructs such a factorization if one exists. The algorithm terminates in a finite number of steps only if the chains $L_{1}, \ldots, L_{n}$ are finite, but the construction behind the algorithm works for infinite bounded chains as well. Therefore we state the main result of this section (Theorem 7) without the finiteness assumption, allowing the algorithm to perform infinitely many steps to produce the desired output. However, we will need to make the additional assumption that the chain $L$ is complete, i.e., that every subset $S \subseteq L$ has an infimum (denoted by $\bigwedge S$ ) and a supremum (denoted by $\bigvee S$ ). Clearly, every finite chain and every closed real interval is complete. 


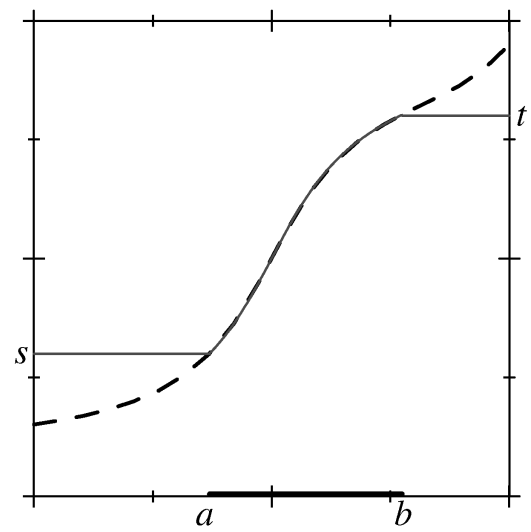

FiguRE 2. The graph of $\varphi_{1}$ as seen through a window

To ensure that the algorithm works correctly, we will also need two reasonable assumptions on the function $f$. The first is that $f$ has no inessential variables, i.e., it depends on all of its variables. If this is not the case, e.g., $f$ does not depend on its first variable, then there is a function $g: L_{2} \times \cdots \times L_{n} \rightarrow L$ such that $f\left(x_{1}, \ldots, x_{n}\right)=$ $g\left(x_{2}, \ldots, x_{n}\right)$. Thus we can apply the algorithm to the function $g$ instead of $f$ (if $g$ still has inessential variables, then we can eliminate them in a similar way). The second assumption is that

$$
f(\mathbf{0})=0 \text { and } f(\mathbf{1})=1 .
$$

If this condition is not met, then the parts of $L$ that lie outside the interval $[f(\mathbf{0}), f(\mathbf{1})]$ are negligible; we may remove them without changing the problem. However, we do not need the assumption that the local utility functions $\varphi_{i}$ share the same range $\mathcal{R}$.

In Subsection 4.1 we first give the intuitive idea behind our construction, and then present Algorithm 1 (Sugeno Utility Function Factorization or SUFF for short). We work out an example in Subsection 4.2 and in Subsection 4.3 we prove the correctness of algorithm SUFF.

4.1. The algorithm SUFF. To present the basic idea of our algorithm, let us suppose that $f(\mathbf{x})=q\left(\varphi_{1}\left(x_{1}\right), \ldots, \varphi_{n}\left(x_{n}\right)\right)$ is a Sugeno utility function, and let us try to extract information about the local utility functions $\varphi_{k}$ from the overall utility function $f$. For notational simplicity, we consider only the case $k=1$; the other cases can be treated similarly. In this case, the pseudo-median decomposition formula (6) takes the form

$$
f\left(x_{1}, x_{2}, \ldots, x_{n}\right)=\operatorname{med}\left(f\left(0, x_{2}, \ldots, x_{n}\right), \varphi_{1}\left(x_{1}\right), f\left(1, x_{2}, \ldots, x_{n}\right)\right) .
$$

By fixing the variables $x_{2}, \ldots, x_{n}$, the left hand side becomes a unary function in the variable $x_{1}$, and on the right hand side we have the median of $\varphi_{1}\left(x_{1}\right)$ and the two constants $s=f\left(0, x_{2}, \ldots, x_{n}\right), t=f\left(1, x_{2}, \ldots, x_{n}\right)$.

Figure 2 depicts this situation, where $L_{1}$ and $L$ are chosen to be the unit interval $[0,1] \subseteq \mathbb{R}$, and the graphs of $f\left(x_{1}, x_{2}, \ldots, x_{n}\right)$ and $\varphi_{1}\left(x_{1}\right)$ are represented by solid and dashed curves, respectively. Observe that these two curves coincide on the interval $] a, b\left[=\left\{x_{1} \in L_{1}: s<f\left(x_{1}, x_{2}, \ldots, x_{n}\right)<t\right\}\right.$, in other words, we can see some part of the graph of $\varphi_{1}$ through the "window" $] a, b[$. To the left of this window $s$ gives an upper bound for $\varphi_{1}\left(x_{1}\right)$, while on the right hand side of the window $t$ gives a lower bound. By fixing the variables $x_{2}, \ldots, x_{n}$ to some other values, we may open other windows which may expose other parts of the graph of $\varphi_{1}$. If we could find sufficiently many windows, then we could recover $\varphi_{1}$. Unfortunately, this is not 
always the case. In fact, as we shall see in the example of Subsection 4.2, the local utility functions are not always uniquely determined by $f$.

For any given $x_{1} \in L_{1}$, let us collect the tuples $\left(x_{2}, \ldots, x_{n}\right)$ that open a window to $\varphi_{1}\left(x_{1}\right)$ into the set $\mathcal{W}_{x_{1}}$. Similarly, let $\mathcal{L}_{x_{1}}$ and $\mathcal{U}_{x_{1}}$ be the sets of tuples that provide only lower and upper bounds, respectively, and let $\mathcal{E}_{x_{1}}$ contain the remaining tuples of $L_{2} \times \cdots \times L_{n}$ :

$$
\begin{aligned}
\mathcal{W}_{x_{1}} & =\left\{\left(x_{2}, \ldots, x_{n}\right): f\left(0, x_{2}, \ldots, x_{n}\right)<f\left(x_{1}, x_{2}, \ldots, x_{n}\right)<f\left(1, x_{2}, \ldots, x_{n}\right)\right\}, \\
\mathcal{L}_{x_{1}} & =\left\{\left(x_{2}, \ldots, x_{n}\right): f\left(0, x_{2}, \ldots, x_{n}\right)<f\left(x_{1}, x_{2}, \ldots, x_{n}\right)=f\left(1, x_{2}, \ldots, x_{n}\right)\right\}, \\
\mathcal{U}_{x_{1}} & =\left\{\left(x_{2}, \ldots, x_{n}\right): f\left(0, x_{2}, \ldots, x_{n}\right)=f\left(x_{1}, x_{2}, \ldots, x_{n}\right)<f\left(1, x_{2}, \ldots, x_{n}\right)\right\}, \\
\mathcal{E}_{x_{1}} & =\left\{\left(x_{2}, \ldots, x_{n}\right): f\left(0, x_{2}, \ldots, x_{n}\right)=f\left(x_{1}, x_{2}, \ldots, x_{n}\right)=f\left(1, x_{2}, \ldots, x_{n}\right)\right\} .
\end{aligned}
$$

Observe that $\mathcal{E}_{x_{1}}$ bears no information on $x_{1}$; we only introduce it for notational convenience. Furthermore, let us define the sets $\mathcal{W}_{x_{1}}^{f}, \mathcal{L}_{x_{1}}^{f}, \mathcal{U}_{x_{1}}^{f}$ as follows:

$$
\begin{aligned}
\mathcal{W}_{x_{1}}^{f} & =\left\{f\left(x_{1}, x_{2}, \ldots, x_{n}\right):\left(x_{2}, \ldots, x_{n}\right) \in \mathcal{W}_{x_{1}}\right\}, \\
\mathcal{L}_{x_{1}}^{f} & =\left\{f\left(x_{1}, x_{2}, \ldots, x_{n}\right):\left(x_{2}, \ldots, x_{n}\right) \in \mathcal{L}_{x_{1}}\right\}, \\
\mathcal{U}_{x_{1}}^{f} & =\left\{f\left(x_{1}, x_{2}, \ldots, x_{n}\right):\left(x_{2}, \ldots, x_{n}\right) \in \mathcal{U}_{x_{1}}\right\} .
\end{aligned}
$$

Note that $\mathcal{W}_{x_{1}}^{f}$ cannot have more than one element, for otherwise $f$ is not a Sugeno utility function. If $\mathcal{W}_{x_{1}}^{f}$ is a one-element set, then let $w_{x_{1}}$ denote its unique element:

$$
w_{x_{1}}=f\left(x_{1}, x_{2}, \ldots, x_{n}\right) \text { if }\left(x_{2}, \ldots, x_{n}\right) \in \mathcal{W}_{x_{1}} .
$$

Furthermore, let $l_{x_{1}}$ and $u_{x_{1}}$ be given as follows:

$$
\begin{gathered}
l_{x_{1}}=\bigvee \mathcal{L}_{x_{1}}^{f} \text { if } \mathcal{L}_{x_{1}} \neq \emptyset \\
u_{x_{1}}=\bigwedge \mathcal{U}_{x_{1}}^{f} \text { if } \mathcal{U}_{x_{1}} \neq \emptyset
\end{gathered}
$$

If any of the sets $\mathcal{W}_{x_{1}}, \mathcal{L}_{x_{1}}, \mathcal{U}_{x_{1}}$ is empty, then the corresponding values $w_{x_{1}}, l_{x_{1}}, u_{x_{1}}$ are undefined. From the above considerations it is clear that $\varphi_{1}$ satisfies the (in)equalities

$$
\varphi_{1}\left(x_{1}\right)=w_{x_{1}}, \varphi_{1}\left(x_{1}\right) \geq l_{x_{1}}, \varphi_{1}\left(x_{1}\right) \leq u_{x_{1}},
$$

whenever the right hand sides are defined.

Let us define a function $\varphi_{1}^{f}: L_{1} \rightarrow L$ by making use of the following four rules:

$(\mathrm{W})$ if $\mathcal{W}_{x_{1}} \neq \emptyset$ then let $\varphi_{1}^{f}\left(x_{1}\right)=w_{x_{1}}$;

(L) if $\mathcal{W}_{x_{1}}=\emptyset, \mathcal{L}_{x_{1}} \neq \emptyset, \mathcal{U}_{x_{1}}=\emptyset$ then let $\varphi_{1}^{f}\left(x_{1}\right)=l_{x_{1}}$;

(U) if $\mathcal{W}_{x_{1}}=\emptyset, \mathcal{L}_{x_{1}}=\emptyset, \mathcal{U}_{x_{1}} \neq \emptyset$ then let $\varphi_{1}^{f}\left(x_{1}\right)=u_{x_{1}}$;

$(\mathrm{LU})$ if $\mathcal{W}_{x_{1}}=\emptyset, \mathcal{L}_{x_{1}} \neq \emptyset, \mathcal{U}_{x_{1}} \neq \emptyset$ then let $\varphi_{1}^{f}\left(x_{1}\right)=l_{x_{1}}$.

Observe that the four cases above cover all possibilities since $\mathcal{W}_{x_{1}}=\mathcal{U}_{x_{1}}=\mathcal{L}_{x_{1}}=\emptyset$ is ruled out by the assumption that $f$ depends on its first variable. It is important to note that $\varphi_{1}^{f}$ is computed only from $f$, without reference to $\varphi_{1}$.

We can define functions $\varphi_{k}^{f}: L_{k} \rightarrow L$ for each $k \in[n]$ in a similar manner, and we will prove that if $f$ is a Sugeno utility function, then these are local utility functions and they provide a factorization $f(\mathbf{x})=q^{f}\left(\varphi_{1}^{f}\left(x_{1}\right), \ldots, \varphi_{n}^{f}\left(x_{n}\right)\right)$, where $q^{f}$ is the Sugeno integral given in Theorem 5 .

$$
q^{f}\left(y_{1}, \ldots, y_{n}\right)=\bigvee_{I \subseteq[n]}\left(f\left(\mathbf{e}_{I}\right) \wedge \bigwedge_{i \in I} y_{i}\right) .
$$

Note that 14 implies that the polynomial $q^{f}$ is indeed a Sugeno integral.

Algorithm 1, which will be referred to as algorithm SUFF in the sequel, summarizes the construction of the local utility functions $\varphi_{k}^{f}$ and the Sugeno integral $q^{f}$. The value false is returned if 


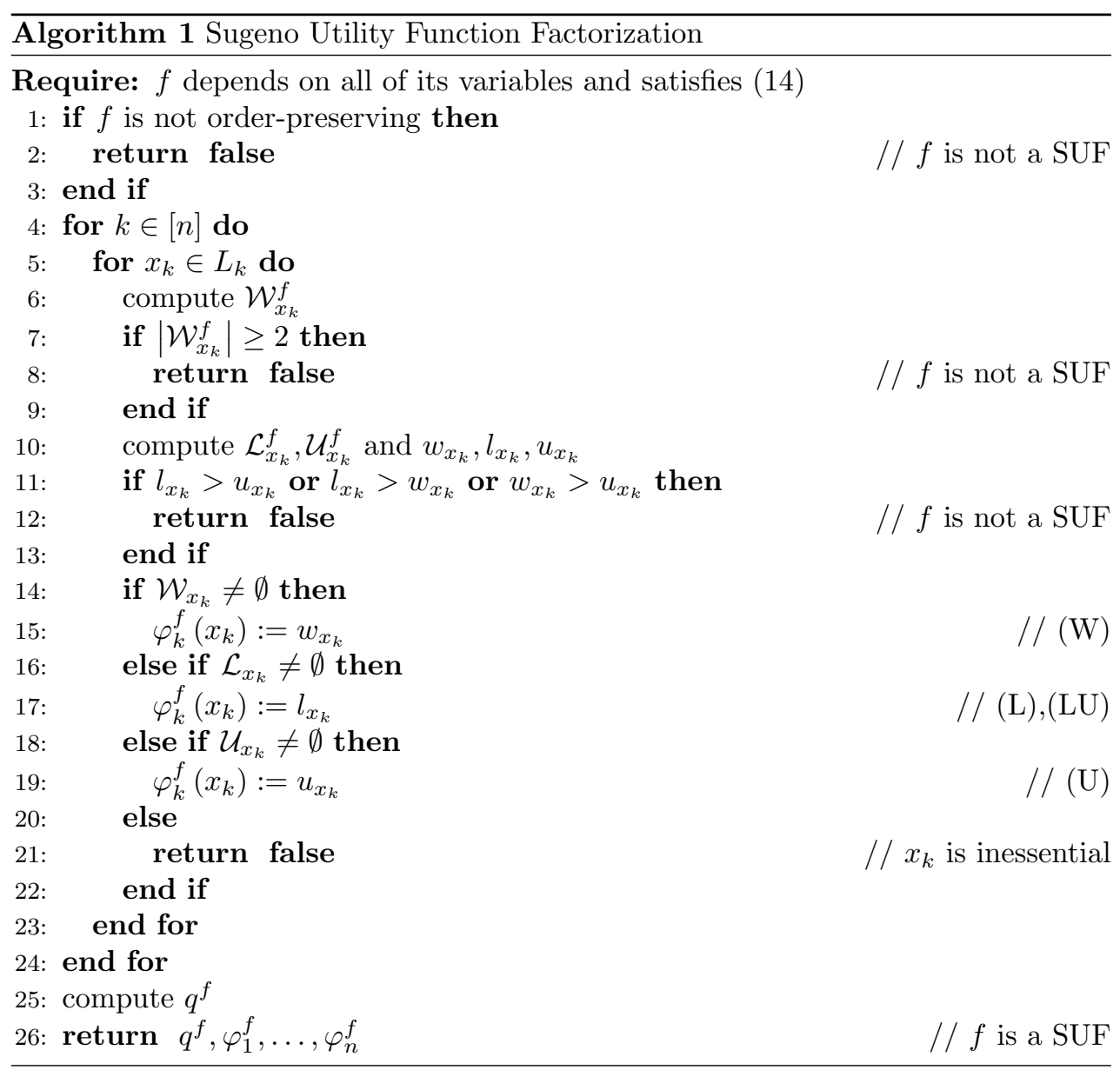

- $f$ is not order-preserving (line 2,

- several different values for $\varphi_{k}\left(x_{k}\right)$ are seen through some windows (line 8),

- the values $l_{x_{1}}, w_{x_{1}}, u_{x_{1}}$ are contradictory (line 12 ), or

- $f$ does not depend on all of its variables (line 21).

Otherwise the output is $q^{f}$ and $\varphi_{k}^{f}(k \in[n])$, which are computed as explained above.

In the next subsection we will prove the following theorem, which ensures the correctness of algorithm SUFF.

Theorem 7. If $f: \prod_{i \in[n]} L_{i} \rightarrow L$ is an order-preserving pseudo-Sugeno integral, then algorithm SUFF constructs a Sugeno integral $q^{f}$ and local utility functions $\varphi_{1}^{f}, \ldots, \varphi_{n}^{f}$ such that

$$
f(\mathbf{x})=q^{f}\left(\varphi_{1}^{f}\left(x_{1}\right), \ldots, \varphi_{n}^{f}\left(x_{n}\right)\right) .
$$

Otherwise, the algorithm outputs the value false.

It is clear that every Sugeno utility function is an order-preserving pseudo-Sugeno integral. Conversely, if $f$ is an order-preserving pseudo-Sugeno integral, then the algorithm SUFF produces a factorization of $f$ into a composition of a Sugeno integral and local utility functions by Theorem 7. Thus $f$ is a Sugeno utility function.

Corollary 2. The class of Sugeno utility functions coincides with the class of orderpreserving pseudo-Sugeno integrals.

Note that the same Sugeno utility function can have several different factorizations, hence starting with a function $f(\mathbf{x})=q\left(\varphi_{1}\left(x_{1}\right), \ldots, \varphi_{n}\left(x_{n}\right)\right)$, just as we did 
TABLE 1. Hotel example: the overall utility function

\begin{tabular}{|c|c|c|c|}
\hline service & price & location & $f$ \\
\hline$*$ & - & $\mathrm{n}$ & 1 \\
\hline$* *$ & - & $\mathrm{n}$ & 2 \\
\hline$* * *$ & - & $\mathrm{n}$ & 2 \\
\hline$* * * *$ & - & $\mathrm{n}$ & 2 \\
\hline$*$ & 0 & $\mathrm{n}$ & 2 \\
\hline$* *$ & 0 & $\mathrm{n}$ & 2 \\
\hline$* * *$ & 0 & $\mathrm{n}$ & 2 \\
\hline$* * * *$ & 0 & $\mathrm{n}$ & 2 \\
\hline$*$ & + & $\mathrm{n}$ & 2 \\
\hline$* *$ & + & $\mathrm{n}$ & 2 \\
\hline$* * *$ & + & $\mathrm{n}$ & 2 \\
\hline$* * * *$ & + & $\mathrm{n}$ & 2 \\
\hline$*$ & - & $\mathrm{y}$ & 3 \\
\hline$* *$ & - & $\mathrm{y}$ & 3 \\
\hline$* * *$ & - & $\mathrm{y}$ & 7 \\
\hline$* * * *$ & - & $\mathrm{y}$ & 8 \\
\hline$*$ & 0 & $\mathrm{y}$ & 5 \\
\hline$* *$ & 0 & $\mathrm{y}$ & 5 \\
\hline$* * *$ & 0 & $\mathrm{y}$ & 7 \\
\hline$* * * *$ & 0 & $\mathrm{y}$ & 8 \\
\hline$*$ & + & $\mathrm{y}$ & 6 \\
\hline$* *$ & + & $\mathrm{y}$ & 6 \\
\hline$* * *$ & + & $\mathrm{y}$ & 7 \\
\hline$* * * *$ & + & $\mathrm{y}$ & 8 \\
\hline
\end{tabular}

at the beginning of this subsection, the factorization $f(\mathbf{x})=q^{f}\left(\varphi_{1}^{f}\left(x_{1}\right), \ldots, \varphi_{n}^{f}\left(x_{n}\right)\right)$ provided by the algorithm may be a different one (see the example in the next subsection).

4.2. An example. Let us illustrate our construction with a concrete (albeit fictitious) example. Customers evaluate hotels along three criteria, namely quality of services, price, and whether the hotel has a good location. Service is evaluated on a four-level scale $L_{1}: *<* *<* * *<* * * *$, price is evaluated on a three-level scale $L_{2}$ : $-<0<+$ (where "-" means expensive, thus less desirable, and "+" means cheap, thus more desirable), and the third scale is $L_{3}: \mathrm{n}(\mathrm{o})<\mathrm{y}$ (es). In addition, each hotel receives an overall rating on the scale $L: 1<\cdots<8$, which gives the overall utility function $f: L_{1} \times L_{2} \times L_{3} \rightarrow L$ (see Table 1). We will find a factorization of this function, and we will analyze its structure in order to draw conclusions about the nature of the "human aggregation" that the customers (unconsciously) perform when forming their opinions about hotels. 
TABLE 2. Hotel example: the partitions of $L_{2} \times L_{3}$

\begin{tabular}{|l||l|l|l|l|}
\hline & \multicolumn{1}{|c|}{$*$} & \multicolumn{1}{|c|}{$* *$} & \multicolumn{1}{|c|}{$* * *$} & \multicolumn{1}{|c|}{${ }^{* * * *}$} \\
\hline \hline$(-, \mathrm{n})$ & $\mathcal{U}_{*}(1)$ & $\mathcal{L}_{* *}(2)$ & $\mathcal{L}_{* * *}(2)$ & $\mathcal{L}_{* * * *}(2)$ \\
\hline$(0, \mathrm{n})$ & $\mathcal{E}_{*}$ & $\mathcal{E}_{* *}$ & $\mathcal{E}_{* * *}$ & $\mathcal{E}_{* * * *}$ \\
\hline$(+, \mathrm{n})$ & $\mathcal{E}_{*}$ & $\mathcal{E}_{* *}$ & $\mathcal{E}_{* * *}$ & $\mathcal{E}_{* * * *}$ \\
\hline$(-, \mathrm{y})$ & $\mathcal{U}_{*}(3)$ & $\mathcal{U}_{* *}(3)$ & $\mathcal{W}_{* * *}(7)$ & $\mathcal{L}_{* * * *}(8)$ \\
\hline$(0, \mathrm{y})$ & $\mathcal{U}_{*}(5)$ & $\mathcal{U}_{* *}(5)$ & $\mathcal{W}_{* * *}(7)$ & $\mathcal{L}_{* * *}(8)$ \\
\hline$(+, \mathrm{y})$ & $\mathcal{U}_{*}(6)$ & $\mathcal{U}_{* *}(6)$ & $\mathcal{W}_{* * *}(7)$ & $\mathcal{L}_{* * * *}(8)$ \\
\hline
\end{tabular}

TABLE 3. Hotel example: the local utility functions

\begin{tabular}{|c||c|c|c|c|}
\hline & $l$ & $w$ & $u$ & $\varphi_{1}^{f}$ \\
\hline \hline$*$ & & & 1 & 1 \\
\hline$* *$ & 2 & & 3 & 2 \\
\hline$* * *$ & 2 & 7 & & 7 \\
\hline$* * * *$ & 8 & & & 8 \\
\hline
\end{tabular}

\begin{tabular}{|c||c|c|c|c|}
\hline & $l$ & $w$ & $u$ & $\varphi_{2}^{f}$ \\
\hline \hline- & & & 1 & 1 \\
\hline 0 & 2 & 5 & & 5 \\
\hline+ & 6 & & & 6 \\
\hline
\end{tabular}

\begin{tabular}{|c||c|c|c|c|}
\hline & $l$ & $w$ & $u$ & $\varphi_{3}^{f}$ \\
\hline \hline $\mathrm{n}$ & & & 1 & 1 \\
\hline $\mathrm{y}$ & 8 & & & 8 \\
\hline
\end{tabular}

First we apply Theorem 5 to find the underlying Sugeno integral:

$$
\begin{aligned}
q^{f}\left(y_{1}, y_{2}, y_{3}\right)= & 1 \vee\left(2 \wedge y_{1}\right) \vee\left(2 \wedge y_{2}\right) \vee\left(3 \wedge y_{3}\right) \\
& \vee\left(2 \wedge y_{1} \wedge y_{2}\right) \vee\left(8 \wedge y_{1} \wedge y_{3}\right) \vee\left(6 \wedge y_{2} \wedge y_{3}\right) \vee\left(8 \wedge y_{1} \wedge y_{2} \wedge y_{3}\right) .
\end{aligned}
$$

Since 1 (resp. 8) is the least (resp. greatest) element of $L$, this polynomial function $q^{f}$ is indeed a Sugeno integral. We can simplify $q^{f}$ by cancelling those terms which are absorbed by some other terms in the disjunction:

$$
q^{f}\left(y_{1}, y_{2}, y_{3}\right)=\left(2 \wedge y_{1}\right) \vee\left(2 \wedge y_{2}\right) \vee\left(3 \wedge y_{3}\right) \vee\left(y_{1} \wedge y_{3}\right) \vee\left(6 \wedge y_{2} \wedge y_{3}\right)
$$

We will be able to perform further simplifications after constructing the local utility functions. Table 2 shows the partitions of $L_{2} \times L_{3}=\mathcal{W}_{x_{1}} \cup \mathcal{L}_{x_{1}} \cup \mathcal{U}_{x_{1}} \cup \mathcal{E}_{x_{1}}$ corresponding to the four possible elements $x_{1} \in L_{1}$. The numbers in parentheses are the values of $f\left(x_{1}, x_{2}, x_{3}\right)$ (recall that we do not compute any values for the sets $\left.\mathcal{E}_{x_{1}}\right)$; these are used to compute the numbers $l_{x_{1}}, w_{x_{1}}, u_{x_{1}}$ shown in Table 3 . This table contains these data for all $x_{2} \in L_{2}$ and $x_{3} \in L_{3}$ as well, together with the values of $\varphi_{1}^{f}\left(x_{1}\right), \varphi_{2}^{f}\left(x_{2}\right), \varphi_{3}^{f}\left(x_{3}\right)$. 
Now that we know that the greatest value of $\varphi_{2}^{f}$ is 6 , we can simplify the Sugeno integral $q^{f}$ by replacing $6 \wedge y_{2} \wedge y_{3}$ with $y_{2} \wedge y_{3}$, and "factoring out" $y_{1} \vee y_{2}$ :

$$
\left(3 \wedge y_{3}\right) \vee\left(\left(y_{1} \vee y_{2}\right) \wedge\left(2 \vee y_{3}\right)\right)=\operatorname{med}\left(3 \wedge y_{3}, y_{1} \vee y_{2}, 2 \vee y_{3}\right)
$$

Note that this polynomial function is different from $q^{f}$, but it gives the same overall utility function $f$. This example shows that the Sugeno integral is not uniquely determined by $f$, and neither are the local utility functions (e.g., we could have chosen $\varphi_{1}^{f}(* *)=3$ according to Remark 9 .

To better understand the behavior of $f$, let us separate two cases upon the location of the hotel:

$$
\begin{aligned}
f\left(x_{1}, x_{2}, x_{3}\right) & =\operatorname{med}\left(3 \wedge \varphi_{3}^{f}\left(x_{3}\right), \varphi_{1}^{f}\left(x_{1}\right) \vee \varphi_{2}^{f}\left(x_{2}\right), 2 \vee \varphi_{3}^{f}\left(x_{3}\right)\right) \\
& = \begin{cases}\left(\varphi_{1}^{f}\left(x_{1}\right) \vee \varphi_{2}^{f}\left(x_{2}\right)\right) \vee 3, & \text { if } x_{3}=\mathrm{y}, \\
\left(\varphi_{1}^{f}\left(x_{1}\right) \vee \varphi_{2}^{f}\left(x_{2}\right)\right) \wedge 2, & \text { if } x_{3}=\mathrm{n} .\end{cases}
\end{aligned}
$$

We can see from 19 that once $x_{3}$ is fixed, what matters is the higher one of $\varphi_{1}^{f}\left(x_{1}\right)$ and $\varphi_{2}^{f}\left(x_{2}\right)$. Thus, instead of aiming at an average level in both, a better strategy would be to maximize one of them. Moreover, $\varphi_{1}^{f}$ either outputs very low or very high scores, whereas $\varphi_{2}^{f}$ is almost maximized once the price is not very bad. Hence it seems more reasonable to focus on service rather than on price. The third variable can radically change the final outcome, but little can be done to improve the location of the hotel.

4.3. Proof of correctness. We assume that $L_{1}, \ldots, L_{n}, L$ are bounded chains, $L$ is complete, $f: \prod_{i \in[n]} L_{i} \rightarrow L$ depends on all of its variables and satisfies (14). If the output of algorithm SUFF is not false, then it computes a Sugeno integral $q^{f}$ and functions $\varphi_{k}^{f}: L_{k} \rightarrow L$ for each $k \in[n]$. It is clear from the construction that

$$
\varphi_{k}^{f}\left(x_{k}\right)=w_{x_{k}}, \varphi_{k}^{f}\left(x_{k}\right) \geq l_{x_{k}}, \varphi_{k}^{f}\left(x_{k}\right) \leq u_{x_{k}}
$$

holds for all $k \in[n], x_{k} \in L_{k}$ (whenever the values on the right hand sides are defined). To prove Theorem 7 we shall make use of two auxiliary lemmas. The first states that the functions $\varphi_{k}^{f}$ are local utility functions, i.e., order-preserving functions.

Lemma 5. If algorithm SUFF does not return the value false, then the functions $\varphi_{1}^{f}, \ldots, \varphi_{n}^{f}$ constructed by the algorithm are local utility functions.

Proof. We show that $\varphi_{1}^{f}$ is order-preserving; the other cases can be treated similarly. Let $a, b \in L_{1}$ such that $a \leq b$. Assume first that $\mathcal{W}_{a} \neq \emptyset$, and fix an arbitrary $\left(x_{2}, \ldots, x_{n}\right) \in \mathcal{W}_{a}$. Then $\varphi_{1}^{f}(a)=w_{a}=f\left(a, x_{2}, \ldots, x_{n}\right)$, and since $f$ is orderpreserving, by the definition of $\mathcal{W}_{a}$, it follows that

$$
f\left(0, x_{2}, \ldots, x_{n}\right)<f\left(a, x_{2}, \ldots, x_{n}\right) \leq f\left(b, x_{2}, \ldots, x_{n}\right) \leq f\left(1, x_{2}, \ldots, x_{n}\right) .
$$

If $f\left(b, x_{2}, \ldots, x_{n}\right)<f\left(1, x_{2}, \ldots, x_{n}\right)$ then $\left(x_{2}, \ldots, x_{n}\right) \in \mathcal{W}_{b}$, hence, by (20) and 15 we have $\varphi_{1}^{f}(b)=w_{b}=f\left(b, x_{2}, \ldots, x_{n}\right)$. If $f\left(b, x_{2}, \ldots, x_{n}\right)=f\left(1, x_{2}, \ldots, x_{n}\right)$, then $\left(x_{2}, \ldots, x_{n}\right) \in \mathcal{L}_{b}$, therefore we have $\varphi_{1}^{f}(b) \geq l_{b} \geq f\left(b, x_{2}, \ldots, x_{n}\right)$ by 20 and (16). In both cases we obtain that

$$
\varphi_{1}^{f}(a)=w_{a}=f\left(a, x_{2}, \ldots, x_{n}\right) \leq f\left(b, x_{2}, \ldots, x_{n}\right) \leq \varphi_{1}^{f}(b),
$$

since $f$ is order-preserving.

The case $\mathcal{W}_{b} \neq \emptyset$ can be treated similarly. Let us now consider the remaining case $\mathcal{W}_{a}=\mathcal{W}_{b}=\emptyset$. Then

$$
\mathcal{L}_{a} \cup \mathcal{U}_{a}=L_{2} \times \cdots \times L_{n} \backslash \mathcal{E}_{a}=L_{2} \times \cdots \times L_{n} \backslash \mathcal{E}_{b}=\mathcal{L}_{b} \cup \mathcal{U}_{b}
$$


Furthermore, from $a \leq b$ we can conclude that $\mathcal{L}_{a} \subseteq \mathcal{L}_{b}$ and $\mathcal{U}_{a} \supseteq \mathcal{U}_{b}$ by making use of the fact that $f$ is order-preserving. This implies that either $\mathcal{L}_{a} \subset \mathcal{L}_{b}$ and $\mathcal{U}_{a} \supset \mathcal{U}_{b}$, or $\mathcal{L}_{a}=\mathcal{L}_{b}$ and $\mathcal{U}_{a}=\mathcal{U}_{b}$. In the first case, by choosing an arbitrary $\left(x_{2}, \ldots, x_{n}\right) \in \mathcal{L}_{b} \backslash \mathcal{L}_{a}=\mathcal{U}_{a} \backslash \mathcal{U}_{b}$ we obtain the desired inequality with the help of (16), (17) and 20):

$$
\varphi_{1}^{f}(a) \leq u_{a} \leq f\left(a, x_{2}, \ldots, x_{n}\right) \leq f\left(b, x_{2}, \ldots, x_{n}\right) \leq l_{b} \leq \varphi_{1}^{f}(b) .
$$

In the second case, we claim that $f\left(a, x_{2}, \ldots, x_{n}\right)=f\left(b, x_{2}, \ldots, x_{n}\right)$ for all $\left(x_{2}, \ldots, x_{n}\right) \in L_{2} \times \cdots \times L_{n}$. This is clear if $\left(x_{2}, \ldots, x_{n}\right) \in \mathcal{E}_{a}=\mathcal{E}_{b}$. If $\left(x_{2}, \ldots, x_{n}\right) \in$ $\mathcal{L}_{a}=\mathcal{L}_{b}$, then

$$
f\left(a, x_{2}, \ldots, x_{n}\right)=f\left(1, x_{2}, \ldots, x_{n}\right)=f\left(b, x_{2}, \ldots, x_{n}\right) .
$$

If $\left(x_{2}, \ldots, x_{n}\right) \in \mathcal{U}_{a}=\mathcal{U}_{b}$, then

$$
f\left(a, x_{2}, \ldots, x_{n}\right)=f\left(0, x_{2}, \ldots, x_{n}\right)=f\left(b, x_{2}, \ldots, x_{n}\right) .
$$

Thus $\mathcal{L}_{a}^{f}=\mathcal{L}_{b}^{f}$ and $\mathcal{U}_{a}^{f}=\mathcal{U}_{b}^{f}$, hence $l_{a}=l_{b}$ and $u_{a}=u_{b}$ (whenever they are defined). Therefore $\varphi_{1}^{f}(a)$ and $\varphi_{1}^{f}(b)$ coincide, no matter which rule (L),(U) or (LU) was used to compute their values.

Remark 9. We can see from the proof of the above lemma that (LU) can be relaxed: $\varphi_{1}^{f}\left(x_{1}\right)$ could be chosen to be any element of the interval $\left[l_{x_{1}}, u_{x_{1}}\right]$ with the convention that whenever we encounter the same interval for different values of $x_{1}$, we always choose the same element of this interval. This guarantees that $\varphi_{1}^{f}$ will be orderpreserving. The proof of Lemma 6 below works with this relaxed rule, since it relies only on the fact that $\varphi_{1}^{f}\left(x_{1}\right) \in\left[l_{x_{1}}, u_{x_{1}}\right]$ whenever $\varphi_{1}^{f}\left(x_{1}\right)$ is determined by rule the $(\mathrm{LU})$.

Lemma 6. Algorithm SUFF does not return the value false if and only if $f$ is an order-preserving pseudo-Sugeno integral. In this case $f$ is pseudo-median decomposable w.r.t. $\varphi_{1}^{f}, \ldots, \varphi_{n}^{f}$.

Proof. For the sufficiency, let us suppose that $f(\mathbf{x})=q\left(\varphi_{1}\left(x_{1}\right), \ldots, \varphi_{n}\left(x_{n}\right)\right)$ is an order-preserving pseudo-Sugeno integral, where $q$ is a Sugeno integral and each $\varphi_{i}$ satisfies the boundary conditions. Clearly, algorithm SUFF will not return false in line 2. Let us note that in the considerations of Subsection 4.1 we did not make use of the fact that each $\varphi_{k}$ is order-preserving, only the order-preservation of $f$, and the pseudo-median decomposition was used. Since the latter holds for pseudo-Sugeno integrals, the observations in Subsection 4.1 still hold for $f$. In particular, 18 holds for $f$, and this means that the algorithm will not return false in lines 8 and 12 . Finally, since $f$ is assumed to depend on all of its variables, line 21 will not return false either.

For the necessity, let us assume that algorithm SUFF does not return false. Then $f$ is clearly order-preserving, and by Lemma 5 , the functions $\varphi_{1}^{f}, \ldots, \varphi_{n}^{f}$ are also order-preserving (hence they satisfy the boundary conditions). By Theorem 4 to prove that $f$ is a pseudo-Sugeno integral it suffices to show that $f$ is pseudo-median decomposable w.r.t. $\varphi_{1}^{f}, \ldots, \varphi_{n}^{f}$. As in the proof of the previous lemma, we focus on the first variable.

We need to show that

$$
\operatorname{med}\left(f\left(0, x_{2}, \ldots, x_{n}\right), \varphi_{1}^{f}\left(x_{1}\right), f\left(1, x_{2}, \ldots, x_{n}\right)\right)=f\left(x_{1}, x_{2}, \ldots, x_{n}\right) .
$$

holds identically. We separate four cases with respect to the partition $L_{2} \times \cdots \times L_{n}=$ $\mathcal{W}_{x_{1}} \cup \mathcal{L}_{x_{1}} \cup \mathcal{U}_{x_{1}} \cup \mathcal{E}_{x_{1}}$.

If $\left(x_{2}, \ldots, x_{n}\right) \in \mathcal{W}_{x_{1}}$, then $\varphi_{1}^{f}\left(x_{1}\right)=w_{x_{1}}=f\left(x_{1}, x_{2}, \ldots, x_{n}\right)$ and

$$
f\left(0, x_{2}, \ldots, x_{n}\right)<f\left(x_{1}, x_{2}, \ldots, x_{n}\right)<f\left(1, x_{2}, \ldots, x_{n}\right) .
$$


Therefore $f\left(0, x_{2}, \ldots, x_{n}\right)<\varphi_{1}^{f}\left(x_{1}\right)<f\left(1, x_{2}, \ldots, x_{n}\right)$, hence the left hand side of 21 is $\varphi_{1}^{f}\left(x_{1}\right)=f\left(x_{1}, x_{2}, \ldots, x_{n}\right)$.

If $\left(x_{2}, \ldots, x_{n}\right) \in \mathcal{L}_{x_{1}}$, then $\varphi_{1}^{f}\left(x_{1}\right) \geq l_{x_{1}}$ according to 20 . Then by 16 and by the definition of $\mathcal{L}_{x_{1}}$ we get

$$
\varphi_{1}^{f}\left(x_{1}\right) \geq l_{x_{1}} \geq f\left(x_{1}, x_{2}, \ldots, x_{n}\right)=f\left(1, x_{2}, \ldots, x_{n}\right) .
$$

Therefore, both sides of (21) are equal to $f\left(1, x_{2}, \ldots, x_{n}\right)$.

The case $\left(x_{2}, \ldots, x_{n}\right) \in \mathcal{U}_{x_{1}}$ follows similarly. Finally, if $\left(x_{2}, \ldots, x_{n}\right) \in \mathcal{E}_{x_{1}}$, then

$$
f\left(0, x_{2}, \ldots, x_{n}\right)=f\left(x_{1}, x_{2}, \ldots, x_{n}\right)=f\left(1, x_{2}, \ldots, x_{n}\right),
$$

hence 21 holds independently of the value of $\varphi_{1}^{f}\left(x_{1}\right)$.

Lemmas 5 and 6 together with Theorem 5 immediately yield Theorem 7

\section{CONCluding REMARKS AND FUture WORK}

Let $X$ be a finite set of alternatives, and let $\preceq$ be a preference relation on $X$ so that $a \preceq b$ reads " $b$ is at least as preferable as $a$ ". In real-life applications, it may be useful to model such preference relations by means of utility functions $\varphi: X \rightarrow L$ where $L$ is a chain ordered by $\leq$ : set $a \preceq b$ if $\varphi(a) \leq \varphi(b)$. If such a utility function exists, then $\preceq$ is a weak order, i.e., reflexive, transitive, and complete. Moreover, it is not difficult to verify that two utility functions $\varphi: X \rightarrow L$ and $\varphi^{\prime}: X \rightarrow L^{\prime}$ induce the same preference relation $\preceq$ if and only if there exists an order-preserving injection $\psi: \operatorname{ran}(\varphi) \rightarrow \operatorname{ran}\left(\varphi^{\prime}\right)$ which satisfies $\psi(\varphi(x))=\varphi^{\prime}(x)$ for all $x \in X$. For further background, see e.g. [3, 15].

In our setting, preference relations are considered on a product $X=\prod_{i \in[n]} L_{i}$ of chains $L_{i}, i \in[n]$, and modeled by Sugeno utility functions $f=q\left(\varphi_{1}, \ldots, \varphi_{n}\right)$ where $q$ is a Sugeno integral on $L$, and each $\varphi_{i}$ is a local utility function which induces a local preference relation $\preceq_{i}$ on $L_{i}$.

Consider the case where $n=2$ and $L_{1}=L_{2}=L=\{0,1\}$. It is not difficult to verify that if the global preference relation is nontrivial and the Sugeno integral $q$ depends on both of its arguments, then the global preference structure uniquely determines the local preference structures and, conversely, the local preference structures uniquely determine the global preference structure.

However, one can easily find examples where the global preference relation $\preceq$ depends on the particular choice of the local utility functions $\varphi_{i}$ and not just on the local preferences $\preceq_{i}$ induced by them. Conversely, the local preference relations are not necessarily determined by the global preference relation. These facts naturally raise the following question: To what extent and in which cases do the local preference relations and the global preference relation determine each other. This question reveals nontrivial problems which constitute a topic of current research.

\section{ACKNOWLEDGMENTS}

We would like to thank Jean-Luc Marichal for useful discussions and for bringing this topic to our attention. The second named author acknowledges that the present project is supported by the TÁMOP-4.2.1/B-09/1/KONV-2010-0005 program of National Development Agency of Hungary, by the Hungarian National Foundation for Scientific Research under grant no. K77409, by the National Research Fund of Luxembourg, and cofunded under the Marie Curie Actions of the European Commission (FP7-COFUND). 


\section{REFERENCES}

[1] G. Beliakov, A. Pradera and T. Calvo, Aggregation Functions: A Guide for Practitioners, Studies in Fuzziness and Soft Computing vol. 221 (Springer, Berlin, 2007).

[2] P. Benvenuti, R. Mesiar and D. Vivona, "Monotone set functions-based integrals", in Handbook of measure theory (North-Holland, Amsterdam, 2002) pp. 1329-1379.

[3] D. Bouyssou, D. Dubois, H. Prade and M. Pirlot (eds), Decision-Making Process - Concepts and Methods (ISTE/John Wiley, 2009).

[4] S. Burris and H. P. Sankappanavar, A Course in Universal Algebra, Graduate Texts in Mathematics vol. 78 (Springer-Verlag, New York, 1981).

[5] M. Couceiro and J.-L. Marichal, "Polynomial Functions over Bounded Distributive Lattices", J. Mult.-Valued Logic Soft Comput. (to appear).

[6] M. Couceiro and J.-L. Marichal, "Characterizations of Discrete Sugeno Integrals as Polynomial Functions over Distributive Lattices", Fuzzy Sets and Systems 161 (5) (2010) 694-707.

[7] M. Couceiro and J.-L. Marichal, "Representations and Characterizations of Polynomial Functions on Chains", J. Mult.-Valued Logic Soft Comput. 16 (1-2) (2010) 65-86.

[8] M. Couceiro and J.-L. Marichal, "Axiomatizations of Quasi-Polynomial Functions on Bounded Chains", Aequationes Math. 396 (1) (2009) 195-213.

[9] M. Couceiro and T. Waldhauser, "Sugeno Utility Functions I: Axiomatizations", Lecture Notes in Artificial Intelligence vol. 6408, (Springer, Berlin, 2010) 79-90.

[10] M. Couceiro and T. Waldhauser, "Sugeno Utility Functions II: Factorizations", Lecture Notes in Artificial Intelligence vol. 6408, (Springer, Berlin, 2010) 91-103.

[11] D. Dubois, J.-L. Marichal, H. Prade, M. Roubens and R. Sabbadin, "The Use of the Discrete Sugeno Integral in Decision-Making: a Survey", Internat. J. Uncertain. Fuzziness KnowledgeBased Systems 9 (5) (2001) 539-561.

[12] J.-C. Fodor and M. Roubens, "Characterization of weighted maximum and some related operations", Inform. Sci. 84 (3-4) (1995) 173-180.

[13] R. L. Goodstein, "The Solution of Equations in a Lattice", Proc. Roy. Soc. Edinburgh Section A $67(1965 / 1967) 231-242$

[14] G. Grätzer, General Lattice Theory (Birkhäuser Verlag, Berlin, 2003).

[15] M. Grabisch, "The application of fuzzy integrals in multicriteria decision making", Eur. J. Oper. Res. 89 (3) (1996) 445-456.

[16] M. Grabisch, J.-L. Marichal, R. Mesiar and E. Pap, Aggregation Functions, Encyclopedia of Mathematics and its Applications vol. 127 (Cambridge University Press, Cambridge, 2009).

[17] M. Grabisch, T. Murofushi and M. Sugeno (eds), Fuzzy Measures and Integrals - Theory and Applications, Studies in Fuzziness and Soft Computing vol. 40 (Physica-Verlag, Heidelberg, 2000).

[18] J.-L. Marichal, "On Sugeno integral as an aggregation function", Fuzzy Sets and Systems 114 (2000) 347-365.

[19] J.-L. Marichal, "Weighted Lattice Polynomials", Discrete Math. 309 (4) (2009) 814-820.

[20] S. Rudeanu, Lattice Functions and Equations, Springer Series in Discrete Mathematics and Theoretical Computer Science (Springer-Verlag, London, 2001).

[21] M. Sugeno, "Theory of fuzzy integrals and its applications", Ph.D. Thesis, Tokyo Institute of Technology, Tokyo, 1974.

[22] M. Sugeno, "Fuzzy measures and fuzzy integrals - a survey", in Fuzzy automata and decision processes, eds. M. M. Gupta, G. N. Saridis, and B. R. Gaines (North-Holland, New York, 1977) pp. $89-102$.

(M. Couceiro) Mathematics Research Unit, FStC, University of Luxembourg, 6, rue Coudenhove-Kalergi, L-1359 Luxembourg, Luxembourg

E-mail address: miguel.couceiro@uni.lu

(T. Waldhauser) Mathematics Research Unit, FStC, University of Luxembourg, 6, rue Coudenhove-Kalergi, L-1359 Luxembourg, Luxembourg, and, Bolyai Institute, University of Szeged, Aradi vértanúk tere 1, H-6720 Szeged, Hungary

E-mail address: twaldha@math.u-szeged.hu 